\title{
Susciter chez les professionnels une prise de conscience critique des enjeux liés à la littératie en santé pour des francophones en situation de minorité linguistique en Ontario
}

\author{
M. S. Zanchetta, Ph. D. (1); C. Maheu, Ph. D. (2); C. Fontaine, B.A. (3); L. Salvador-Watts, B. Sc. inf. (4); \\ N. Wong, B. Sc. inf. (5)
}

Cet article a fait l'objet d'une évaluation par les pairs.

Diffuser cet article sur Twitter

\section{Résumé}

Introduction : Nous avons procédé à une évaluation qualitative des changements immédiats sur les plans de l'apprentissage et de l'attitude chez des professionnels de la santé et des services sociaux ayant participé à un atelier visant à susciter une réflexion critique sur la littératie en santé des Franco-Ontariens en situation de minorité linguistique.

Méthodologie : L'étude a été réalisée auprès de 41 professionnels francophones de la santé et des services sociaux. L'animatrice de l'atelier a utilisé des objets évocateurs pour susciter la réflexion sur la littératie en santé. Les sources de données étaient les enregistrements audio des discussions de groupes et les formulaires de rétroaction remplis par les participants.

Résultats : L'étude a révélé que l'atelier avait suscité chez les participants une prise de conscience à propos de la littératie en santé et les avait incités à promouvoir la littératie en santé dans leur pratique professionnelle. L'atelier a aussi élargi la vision de la littératie en santé qu'avaient les participants à sa dimension de déterminant social de la santé qui agit en synergie avec la culture, l'âge, le statut à l'égard de l'immigration, le soutien social et le statut socioéconomique.

Conclusion : Les professionnels ont estimé que la prise de conscience des problèmes de littératie en santé relevait d'une responsabilité collective. Cela corrobore notre hypothèse selon laquelle une pédagogie critique appliquée à la formation continue stimule chez les professionnels la prise de conscience de leur capacité à vouloir changer leur pratique et leur milieu de travail.

Mots-clés : étude d'évaluation, minorité linguistique francophone, santé des minorités, activités de formation

\section{Introduction}

La littératie en santé (LS) est considérée comme un déterminant social de la santé plus puissant que l'âge, le sexe, le niveau de scolarité, l'origine ethnique, l'emploi et le statut socio-économique ${ }^{1}$. Elle est un prédicteur des connaissances des clients au sujet de leur auto-prise en charge des maladies chroniques ${ }^{2}$. L'auto-prise en charge implique de développer sa capacité à prendre soin de soi-même ainsi que d'améliorer son autonomie, étapes le plus souvent franchies grâce à l'apprentissage participatif, une méthode utilisée par ailleurs dans les programmes d'éducation en santé pour informer les professionnels de la santé au sujet de l'asthme, de l'hypertension, du sida, de la tuberculose et d'autres maladies chroniques ${ }^{3-5}$. Au Canada, l'apprentissage participatif est utilisé pour favoriser l'auto-prise en charge en renforçant la $\mathrm{LS}^{6}$, ce qui est difficile lorsque l'information en santé est présentée dans une autre langue que la langue maternelle ${ }^{7}$.

Dans cet article, nous traitons des attitudes envers la LS des professionnels francophones, nés au Canada ou ailleurs, qui vivent en situation de minorité linguistique en Ontario ainsi que des enjeux associés à la LS que ces professionnels rencontrent en travaillant avec des clients francophones.

Nous définissons comme francophone toute personne de langue maternelle française $^{8}$ capable de converser dans cette langue 9 . L'appartenance à une minorité linguistique francophone embrasse l'identité ethnolinguistique, l'identité sociale et la signification affective de ces identités ${ }^{10}$.

L'Ontario compte la plus forte proportion d'immigrants francophones au Canada après le Québec; 10,3 \% de ces immigrants francophones font partie d'une minorité ethnoculturelle, et, parmi ces derniers,

Rattachement des auteurs :

1. École des sciences infirmières Daphne Cockwell, Faculté des services communautaires, Université Ryerson, Toronto (Ontario), Canada

2. École de sciences infirmières Ingram, Faculté de médecine, Université McGill, Montréal (Québec), Canada

3. Regroupement des intervenants francophones en santé et en services sociaux de l'Ontario (RIFSSSO), Toronto (Ontario), Canada

4. Family Birthing Centre, St. Joseph's Health Centre, Toronto (Ontario), Canada

5. Médecine interne générale, Toronto Western Hospital, Toronto (Ontario), Canada

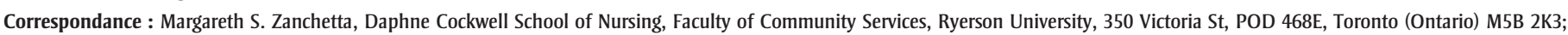
tél. : 416-979-5000, poste 4557; téléc. : 416-979-5332; courriel : mzanchet@ryerson.ca 
$86 \%$ vivent en situation de minorité linguistique ${ }^{11,12}$. L'Ontario compte aussi une forte proportion de francophones « natifs » âgés possédant un faible niveau de littératie générale ${ }^{13}$. On ne dispose pas d'information spécifique sur les professionnels de la santé francophones en Ontario, mais on sait que de manière générale les professionnels de la santé canadiens sont peu conscients de la nature, de l'importance et de l'impact de la $\mathrm{LS}^{14}$ sur leurs clients en situation de minorité linguistique. Nous présentons les conclusions d'une étude d'évaluation d'un atelier réunissant des professionnels de la santé et des services sociaux francophones. Le but de cet atelier était d'une part de connaître et d'évaluer les connaissances de ces professionnels au sujet de la LS des minorités linguistiques francophones auxquelles appartenaient leurs clients et d'autre part de promouvoir les pratiques exemplaires en matière de LS permettant de combler les besoins de leurs clients en matière de santé et de renforcer la bonne volonté dont ces derniers font preuve en désirant prendre leur santé en main.

\section{Contexte}

Une proportion étonnamment forte de Canadiens adultes - $42 \%$ des adultes en âge de travailler (16 à 65 ans) et $56 \%$ des francophones - trouvent le contenu des documents écrits en difficile à lire dans leur langue maternelle ${ }^{13}$. Ce défaut de compréhension diminue la LS et augmente les coûts de santé ${ }^{15,16}$, parce que la motivation à suivre les principes de promotion de la santé nécessite de comprendre l'information en santé ${ }^{17}$. Le lien entre LS et disparités sur le plan de l'accès aux soins de santé des francophones en situation de minorité linguistique au Canada demeure relativement peu connu $^{18}$. La littérature relative à la LS elle-même est également peu abondante pour cette population, tout comme les évaluations des interventions visant à augmenter la LS ou à améliorer la formation continue des professionnels à propos de la LS. Notre étude d'évaluation cherche à combler cette lacune dans les connaissances.

De multiples définitions de la LS ont été formulées, que ce soit dans la perspective des déterminants sociaux de la santé ou dans celle de l'auto-prise en charge des maladies ${ }^{19}$. Dans la perspective des déterminants sociaux de la santé, la LS est définie comme une connaissance construite socialement tout au long de la vie ${ }^{7}$ et qui englobe la lecture, l'écriture, l'écoute, la parole, la numératie et la pensée critique, lesquelles sont fortement influencées par la langue et la culture ${ }^{20}$. La LS influe sur l'état de santé en agissant en synergie avec d'autres déterminants sociaux de la santé (en particulier l'accès aux soins de santé, l'âge, la culture, le lieu de résidence, le niveau de scolarité et le revenu). La LS façonne également les croyances en santé, les décisions en matière de santé, le choix $\mathrm{du}$ mode de $v^{21}{ }^{21}$ et la façon dont on navigue à l'intérieur de systèmes de santé complexes. Une LS faible ou insuffisante contribue donc aux inégalités d'accès aux soins de santée ${ }^{22}$.

Dans la perspective de l'auto-prise en charge des maladies, la LS est définie comme un ensemble de capacités cognitives mesurables (en particulier lire, écrire et compter) qui permettent une compréhension de la santé, une utilisation adéquate des services de santé, la prise de décisions prudentes et l'observance des traitements médicaux et des schémas thérapeutiques $^{23}$. Les mesures de LS sont des outils prédictifs approximatifs des comportements en santé, des résultats en termes de santé, des comportements qui favorisent la santé, des inégalités du système de soins de santé ${ }^{24}$, des consultations aux urgences et des coûts élevés des soins de santé liés à une compréhension insuffisante de l'information médicale et à une prise en charge inadéquate des maladies en général ${ }^{25,26}$. Une LS élevée est associée aussi à une satisfaction à l'égard des services de santé, à un optimisme au sujet des soins et à une confiance envers les systèmes de santé et les professionnels de la santé27.

Nous avons utilisé pour notre étude la définition de la LS formulée dans la perspective des déterminants sociaux de la santé à laquelle nous avons ajouté des principes de communication en santé ${ }^{28}$ et de littératie critique $^{15}$. Dans cette perspective combinée, la LS englobe des processus multidimensionnels d'apprentissage social (en particulier l'accumulation d'acquis familiaux, scolaires, sociaux, culturels et professionnels) qui intègrent les valeurs, les croyances, les craintes et les comportements en lien avec la santé. À l'origine de la LS, on trouve 1) la culture et les connaissances en matière de santé, 2) le type d'éducation en santé à laquelle une personne est soumise, 3) l'habitude de chercher, lire, décoder et communiquer l'information dans le domaine de la santé, 4) la capacité d'utiliser l'information numérique sur la santé pour résoudre des problèmes de santé et 5) l'application d'autres formes de littératie pour décoder le monde ${ }^{7}$. La LS est considérée comme agissant en synergie avec d'autres déterminants au-delà de la volonté et de la capacité d'un individu à l'auto-prise en charge de sa maladie.

\section{Recension des écrits}

L'idée que, selon le principe de la conscientisation critique en éducation de Freire $^{29}$, la littératie critique peut aider les individus à effectuer un changement social a servi de guide aux études d'évaluation des ateliers sur le renouvellement de la pratique destinés aux professionnels de la santé et des services sociaux. La perspective utilisée par Freire aide les professionnels à réfléchir sur leur travail et sur leur institution en tenant compte des contextes politique, socioéconomique et professionnel $^{3}$. Les résultats de ce type d'études montrent un engagement à favoriser l'autonomisation des clients, une meilleure compréhension des clients et une réflexion plus approfondie sur les difficultés de communication $^{30}$. Les principes de conscientisation critique, d'autonomisation, d'émancipation et d'apprentissage participatif appliqués aux professionnels et aux clients orientent les initiatives de promotion de la santé depuis 30 ans $^{31,32}$. Il est bon de souligner que, bien qu'une meilleure LS soit le résultat attendu de ces initiatives de promotion de la santé, ces concepts ne sont ni équivalents ni interchangeables.

\section{Cadre conceptuel}

Dans sa philosophie de l'éducation, Freire définit la " conscience critique ${ }^{29,33}$ comme un état qui permet à l'homme de comprendre son monde personnel et la place qu'il occupe dans le monde, et de 
prendre part à la transformation de ce monde ${ }^{34}$. La conscience critique est un moyen de réflexion au sein du processus d'action-réflexion-action. Le dialogue critique entre participants au cours de l'apprentissage est source de nouvelles connaissances. Freire postule que la conscience acquise par cet apprentissage peut motiver les apprenants à trouver par euxmêmes le meilleur moyen de résoudre leurs problèmes. Autrement dit, grâce à la compréhension de la manière dont leur réalité sociale influe sur leur apprentissage, ils sont mieux à même de reconnaître qu'il faut s'opposer aux conditions d'oppression, envisager d'autres modes de fonctionnement puis se fixer des objectifs d'apprentissage en fonction de son niveau de formation ${ }^{33,35}$.

Les travaux de Freire ont guidé la conception, l'élaboration et l'évaluation de notre atelier sur la LS à l'intention des professionnels francophones. Le principe de " conscience critique » de Freire ${ }^{36}$ était particulièrement bien adapté à l'atelier parce que les participants francophones étaient tous conscients de l'oppression liée à leur statut de minorité linguistique au Canada et de ses racines historiques. Le silence sur cette oppression a mené à une perte d'identité culturelle, sociale, communautaire et ethnique au sein des minorités linguistiques francophones et à une perception de la part des nonfrancophones que parler français est le fait d'une population minoritaire et colonisée ${ }^{37}$.

\section{Méthodologie}

\section{Questions de recherche}

Nous avons effectué une étude d'évaluation de l'atelier «Placez la littératie en santé au cœur de la pratique », destiné aux professionnels francophones de la santé et des services sociaux et qui avait comme objectif de diffuser de l'information empirique au sujet de la LS et d'amorcer une réflexion à propos de la LS des clients de ces professionnels.

Notre évaluation s'est fondée sur les questions suivantes :

- Comment l'atelier a-t-il élargi la vision de la LS chez les participants?
- Sur quels comportements, attitudes, capacités ou motivations l'atelier a-t-il eu une influence?

- Est-ce que les changements concernant la compréhension des clients et la prestation des services par les participants peuvent être attribués à l'atelier?

\section{L'atelier de conscientisation à l'égard de la littératie en santé}

Le but de l'atelier " Placez la littératie en santé au cœur de la pratique » était de révéler les connaissances des professionnels à propos de la LS et de leur faire prendre conscience de deux grands problèmes en lien avec la LS et la santé chez les francophones : le peu de publications et le peu de connaissances des professionnels francophones au sujet des déterminants sociaux de la santé et des besoins particuliers des minorités linguistiques francophones ${ }^{10}$ et le peu de connaissances des professionnels de la santé et des services sociaux canadiens à propos de la $\mathrm{LS}^{14}$. Nous avons adopté une approche constructiviste grâce à laquelle le dialogue social se traduit par un apprentissage fondé sur la réflexion individuelle à propos de ses propres constructions sociales $^{38}$ ainsi qu'à propos de la manière dont la connaissance permet aux individus de poursuivre des buts dans de multiple contextes $^{39}$.

Étant donné qu'il s'agissait d'un atelier de conscientisation, les connaissances des participants n’ont été mesurées ni avant ni après l'atelier. Les preuves d'apprentissage se sont manifestées dans les réflexions des participants et les discussions de groupe ${ }^{31}$. L'atelier a eu lieu dans les trois villes ontariennes où vivent la majorité des professionnels de la santé et des services sociaux francophones : Sudbury (en décembre 2008), Toronto (en janvier 2009) et Ottawa (en février 2009). L'atelier, d'une durée de six heures, a eu lieu une fois dans chaque ville, avec un nombre de participants variant entre 9 et 18.

L’information empirique présentée dans le cadre de l'atelier était constituée de statistiques canadiennes sur les niveaux de littératie et de résultats des recherches sur la LS publiés par le Conseil canadien sur l'apprentissage ${ }^{22}$ et disponibles sur leur site $\mathrm{Web}^{40}$, par le Consortium national de formation en santé pour les populations francophones $^{41}$ et par l'Association canadienne de santé publique et ses chercheurs associés ${ }^{6,14}$. Durant l'atelier, les participants ont été invités à travailler avec des objets évocateurs (photographies, dessins ou objets) qui ont fait progresser le dialogue dialectique entre l'animatrice de l'atelier et les participants $^{42}$ et qui leur ont servi, en premier lieu, à se remémorer leurs expériences et leurs réalités sociales ainsi qu'à prendre conscience de la teneur de leurs idées, en deuxième lieu, à créer des analogies guidant leur réflexion individuelle à propos de leur pratique et afin de la transformer et, en troisième lieu, à réfléchir sur leur expérience personnelle et à décider d'agir en fonction d'elle. Pour assurer l'uniformité d'un atelier à l'autre, l'animatrice (MZ) a choisi les mêmes objets évocateurs lors des trois ateliers.

Le tableau 1 décrit les composantes de l'atelier, fondées sur le concept d'actionréflexion-action de Freire $^{29}$, sur l'utilisation d'objets évocateurs et sur le dialogue critique.

\section{Recrutement des participants à l'atelier et à l'étude}

Afin d'avoir une compréhension approfondie des résultats immédiats de l'atelier, nous avons recueilli des données détaillées au sujet de nos participants au moyen d'une étude d'évaluation qualitative ${ }^{43}$. Le comité d'éthique de la recherche de l’Université York a autorisé la tenue de l'étude. Lorsque les participants y consentaient, nous avons procédé à un enregistrement de leurs propos.

Les participants à l'atelier étaient des professionnels francophones originaires du Burkina Faso, d'Haïti, du Liban, du Maroc et du Canada qui travaillaient dans la santé et les services sociaux (incluant les gestionnaires de projet), l'élaboration des politiques ou la défense des droits des citoyens. Des étudiants dans des disciplines liées à la santé ou aux services sociaux ont aussi participé à l'atelier (voir le tableau 2). 
TABLEAU 1

Description de l'atelier « Placez la littératie en santé au cœur de la pratique »

\begin{tabular}{|c|c|c|c|}
\hline Sujets présentés par l'animatrice & Exercice 1 & Exercice 2 & Évaluation \\
\hline $\begin{array}{l}\text { Données statistiques sur la littératie } \\
\text { et la LS en lien avec le lieu de l'atelier. } \\
\text { Survol des données scientifiques sur la LS. } \\
\text { Revue des définitions conceptuelles } \\
\text { de la LS. } \\
\text { Discussion au sujet des nombreuses } \\
\text { définitions de la LS. } \\
\text { Exercice interactif pour comparer les } \\
\text { compétences fonctionnelles des } \\
\text { personnes généralement alphabètes } \\
\text { et des personnes généralement } \\
\text { analphabètes. } \\
\text { Discussion sur les déterminants } \\
\text { sociaux de la santé liés à une } \\
\text { faible LS au sein de la minorité } \\
\text { linguistique francophone. } \\
\text { Discussion interactive à propos de } \\
\text { ce qu'ont compris les participants } \\
\text { de l'exposé et de leurs expériences } \\
\text { personnelles et professionnelles. }\end{array}$ & $\begin{array}{l}\text { But : Promouvoir l'application } \\
\text { et la compréhension du concept } \\
\text { de LS au moyen de métaphores } \\
\text { et d'analogies. } \\
\text { Formation de groupes de discussion. } \\
\text { Distribution d'objets évocateurs à } \\
\text { tous les participants. } \\
\text { Réflexion individuelle sur l'exposé, } \\
\text { le concept de LS et les idées } \\
\text { évoquées par les objets. } \\
\text { Exposé individuel de chaque } \\
\text { participant sur les métaphores } \\
\text { et analogies évoquées par les objets. } \\
\text { Conclusion de l'exercice par une } \\
\text { synthèse des exposés des } \\
\text { participants par l'animatrice. }\end{array}$ & $\begin{array}{l}\text { But : Provoquer une réflexion critique } \\
\text { au sujet des compétences essentielles } \\
\text { à la LS en milieu professionnel afin } \\
\text { de conscientiser les professionnels } \\
\text { à la LS. } \\
\text { Discussions de groupe au sujet } \\
\text { des compétences essentielles à } \\
\text { l'intégration du concept de LS dans } \\
\text { sa pratique et du soutien nécessaire } \\
\text { à l'échelle de l'organisme et de } \\
\text { la collectivité pour améliorer les } \\
\text { connaissances et les compétences } \\
\text { des professionnels en matière de LS. } \\
\text { Court exposé de chaque groupe au } \\
\text { sujet des besoins répertoriés. } \\
\text { Conclusion de l'exercice par une } \\
\text { synthèse des principales idées } \\
\text { du groupe par l'animatrice. }\end{array}$ & $\begin{array}{l}\text { Distribution du formulaire visant à } \\
\text { obtenir une opinion sur : } \\
\text { - l'évaluation de la mesure dans } \\
\text { laquelle l'atelier a permis aux } \\
\text { participants d'en apprendre } \\
\text { davantage au sujet de la LS; } \\
\text { - la conscience des difficultés qu'ont } \\
\text { les francophones en situation } \\
\text { minoritaire à obtenir des services } \\
\text { en français; } \\
\text { - la conscience de l'importance } \\
\text { d'offrir des services de santé } \\
\text { et des services sociaux adaptés } \\
\text { sur les plans linguistique et culturel; } \\
\text { - des suggestions pour les futurs } \\
\text { ateliers. }\end{array}$ \\
\hline
\end{tabular}

Abréviation : LS, littératie en santé.

Remarque : Nous avons décidé de présenter une description détaillée de l'atelier afin d'informer les lecteurs au sujet de l'utilisation d'objets évocateurs conformément à la méthode pédagogique de Freire $^{29}$ et pour en permettre la répétition.

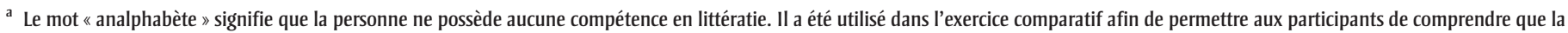
dimension liée au raisonnement en littératie ne dépend pas des compétences en lecture.

TABLEAU 2

Distribution des participants à l'atelier « Placez la littératie en santé au cour de la pratique » par ville et par secteur professionnel

\begin{tabular}{|c|c|c|c|}
\hline Ville & Secteur $^{\mathrm{a}}$ & Profession et champ d'activité & $\mathbf{N}$ \\
\hline \multirow[t]{4}{*}{ Sudbury } & Santé & $\begin{array}{l}\text { Infirmiers autorisés, professeur de physiothérapie, diététiste, } \\
\text { gestionnaire en santé communautaire, organisateurs } \\
\text { communautaires, agent de développement communautaire }\end{array}$ & 7 \\
\hline & Services sociaux & Travailleur social & 1 \\
\hline & Éducation & Professeur de sciences sociales au niveau collégial & 1 \\
\hline & Sous-total & & 9 \\
\hline \multirow[t]{4}{*}{ Toronto } & Santé & $\begin{array}{l}\text { Éducateurs en santé, étudiant de premier cycle en sciences } \\
\text { infirmières, gestionnaire de projet, psychologue, expert-conseil }\end{array}$ & 6 \\
\hline & Services sociaux & $\begin{array}{l}\text { Travailleurs sociaux, travailleurs communautaires, responsable } \\
\text { de l'élaboration de politiques, anthropologue, professionnels } \\
\text { en communication, coordonnateurs de projet, comptable }\end{array}$ & 10 \\
\hline & Éducation & Professeur de sciences sociales au niveau collégial & 1 \\
\hline & Sous-total & & 17 \\
\hline \multirow[t]{4}{*}{ Ottawa } & Santé & $\begin{array}{l}\text { Sage-femme, étudiants en sciences infirmières, médecin, } \\
\text { gestionnaire de projet, défenseur des droits des citoyens, } \\
\text { coordonnateurs de projets, infirmiers autorisés }\end{array}$ & 14 \\
\hline & Services sociaux & Étudiant en sciences sociales au niveau collégial & 1 \\
\hline & Éducation & $\begin{array}{l}\text { Enseignant au niveau secondaire, professeur de sciences } \\
\text { infirmières au niveau universitaire, administrateur d'école }\end{array}$ & 3 \\
\hline & Sous-total & & 18 \\
\hline Total & & & 44 \\
\hline
\end{tabular}

Abréviation : RIFSSSO, Regroupement des intervenants francophones en santé et en services sociaux de l'Ontario.

${ }^{\text {a }}$ Ces secteurs représentent fort probablement les secteurs d'activité professionnelle des membres du RIFSSSO.
Les 44 participants à l'atelier étaient tous membres du Regroupement des intervenants francophones en santé et en services sociaux de l'Ontario (RIFSSSO) et ont été recrutés (par CF) grâce à la liste de diffusion de cet organisme. Nous n'avons utilisé aucun critère de sélection des participants, et la taille de l'échantillon a été déterminée par le nombre de personnes qui ont accepté de participer à l'étude au début de l'atelier. Il a donc été impossible d'obtenir une distribution uniforme reflétant les divers champs de pratique des participants.

Un membre de l'équipe ( $\mathrm{CF}$ ) a expliqué les avantages et les risques associés à la participation à l'étude d'évaluation et le droit des participants à refuser l'enregistrement. Une période de questions a suivi, et les participants ont été invités à lire et à signer le formulaire de consentement éclairé. Sur les 44 participants à l'atelier, 41 ont accepté de participer à l'étude (voir le tableau 2). Lorsque les participants ayant refusé de participer à l'étude ont pris la parole, l'enregistrement a été arrêté. 


\section{Collecte et analyse des données}

Dix-sept heures d'enregistrement ont été transcrites. On a aussi demandé aux participants de remplir un formulaire sur leurs opinions comportant des questions sur la façon dont l'atelier avait contribué à leur faire connaître le concept de LS et à leur faire prendre conscience des problèmes liés à la LS chez leurs clients. Les transcriptions et les formulaires ont été analysés manuellement ${ }^{44}$ de la façon suivante :

1) lecture des transcriptions;

2) regroupement des idées descriptives et des idées explicatives;

3) création de groupes composés de mots et de phrases ayant la même signification;

4) repérage de dichotomies dans les catégories (p. ex. positif/négatif, favorable/défavorable);

5) regroupement des preuves de l'impact de l'apprentissage au sujet de la LS en 3 catégories conceptuelles :

- accroissement des connaissances,

- changements d'attitude,

- changements envisagés dans la pratique professionnelle;

6) caractérisation des thèmes relevés au cours de l'analyse :

- reconnaissance des diverses dimensions de la LS (prévention, auto-prise en charge de la santé, droit d'accès aux services, équité des services, langue comme déterminant social de la santé),

- vision élargie de la santé (environnement, éducation, économie, sexe, réseau social, âge),

- conscientisation critique (niveaux de LS chez les francophones, vie en situation de minorité linguistique);

7) relecture des transcriptions en fonction des thèmes;

8) analyse des associations entre les thèmes;

9) nouvelle analyse des transcriptions pour déterminer s'il y avait eu conscientisation à l'égard de la LS.

MZ, LS et NW, qui parlent très bien le français même si ce n'est pas leur langue maternelle, ont analysé les données. Ensuite, quatre professionnels francophones vivant en situation de minorité linguistique en Ontario ont vérifié les résultats. À titre d'experts francophones, $\mathrm{CM}$ et $\mathrm{CF}$ ont confirmé l'interprétation originale des résultats ${ }^{45}$. Deux autres professionnels francophones possédant une expérience dans les secteurs de la santé et de l'éducation ont relu la première ébauche du manuscrit.

\section{Résultats}

Seuls trois participants avaient des connaissances au sujet de la LS au moment de l'inscription à l'atelier. Après l'exposé de l'animatrice, la plupart des participants à l'étude d'évaluation ont avoué que c'était la première fois qu'ils entendaient parler de LS dans le contexte des services de santé et des services sociaux et de l'importance de la LS pour leurs clients :

C'est la première fois que j'entends parler de littératie en santé. C'est incroyable comme cette littératie fait partie de notre travail quotidien et combien il est important de bien la comprendre afin d'aider la population âgée canadienne. (participant de Sudbury) ${ }^{*}$

Lorsque l'animatrice a utilisé la carte interactive de l'Ontario en ligne du Conseil canadien sur l'apprentissage ${ }^{46}$ pour montrer les niveaux de littératie et de lecture, les participants ont immédiatement fait le rapprochement avec la réalité de la LS de leurs clients. Ils ont discuté de la manière dont une faible littératie nuit à leurs efforts pour améliorer la LS de leurs clients lorsqu'ils ne disposent essentiellement que de matériel écrit.

\section{Idées préliminaires des participants au sujet de la littératie en santé}

Les histoires personnelles et professionnelles que les participants à l'atelier ont racontées pendant l'exercice 1 (voir le tableau 1) témoignaient de leur conscience des conséquences d'une faible LS. L'accès à l'information en santé s'est révélé un enjeu fondamental dans les discussions de groupe. Quatre idées interreliées ont émergé : 1) comment la LS influence la manière dont les clients comprennent l'information en santé et le fait qu'ils la comprennent ou non; 2) l'importance d'écouter les clients; 3) la nécessité pour les professionnels de fournir une information en santé simple et claire et 4) la nécessité pour les professionnels d'aller au-delà d'une présentation superficielle de l'information en santé.

Les objets évocateurs ont fait émerger diverses associations d'idées. Un flacon de vernis à ongles a évoqué les normes de la société à l'égard de la santé des femmes, les soins des ongles, l'image corporelle, le coût des manucures et le choix qu'il faut parfois faire entre se payer de la nourriture ou se payer des produits de beauté; un biberon a évoqué le risque de contamination du lait par des plastiques de mauvaise qualité ainsi qu'une hygiène bucco-dentaire déficiente chez les nourrissons; une boîte de thon en conserve a évoqué la difficulté à lire les petits caractères sur les boîtes de conserve ainsi que les mauvaises interprétations de l'information nutritionnelle (voir d'autres exemples dans le tableau 3).

\section{Réflexion des participants à propos de la littératie en santé et de leurs expériences en lien avec la maladie}

Les participants ont réfléchi sur le degré de compréhension de la probabilité - concept souvent utilisé pour expliquer l'information sur le diagnostic et le pronostic - que pouvait avoir leurs clients à faible niveau de LS. Le manque de matériel éducatif en santé qui puisse atténuer les problèmes de LS et le manque d'aide pour décoder l'information en santé aggravent l'effet d'une faible littératie numérique. Ainsi, les clients préfèrent une information en santé qui provoque moins de stress, par exemple qu'on leur dise que leur probabilité de ne pas développer un cancer est de $95 \%$ plutôt que d'apprendre que leur probabilité de développer un cancer est de $5 \%$. La littératie numérique est nécessaire pour interpréter l'information en santé, par exemple pour calculer les lipides, les glucides et les calories dans un régime alimentaire. Les participants ont aussi souligné que

\footnotetext{
* Il s'agit d'une traduction de la version anglaise approuvée par les auteurs.
} 
TABLEAU 3

Idées suscitées par les objets évocateurs utilisés dans les exercices de l'atelier "Placez la littératie en santé au cœur de la pratique »

\begin{tabular}{|c|c|c|}
\hline $\begin{array}{l}\text { Catégories } \\
\text { conceptuelles }\end{array}$ & Objet évocateur & Idées évoquées par les objets \\
\hline \multirow[t]{2}{*}{$\begin{array}{l}\text { Accroissement des } \\
\text { connaissances }\end{array}$} & $\begin{array}{l}\text { Suce (tétine) } \\
\text { pour bébé }\end{array}$ & $\begin{array}{l}\text { «Enlever les suces [des clients]... l'information peut passer... } \\
\text { lorsque les clients sont différents... nous leur enlevons } \\
\text { [leur suce]... de façon que nous puissions comprendre. » } \\
\text { (participant d'Ottawa) }\end{array}$ \\
\hline & $\begin{array}{l}\text { Boîte à lunch } \\
\text { "Pas de problème » } \\
\text { de Bart Simpson }\end{array}$ & $\begin{array}{l}\text { "Les clients arrivent avec son "no problemo" mais sont } \\
\text { malades... tout son bagage dans la boîte... il va se } \\
\text { remplir, s'aider à lui expliquer ce que la personne a. » } \\
\text { (participant de Toronto) }\end{array}$ \\
\hline \multirow[t]{2}{*}{$\begin{array}{l}\text { Changements } \\
\text { dans la pratique } \\
\text { professionnelle }\end{array}$} & Bonsaï & $\begin{array}{l}\text { "On cherchera des informations avec les racines dans le sol... } \\
\text { on essaie de construire le tronc... il faut renouveler ses } \\
\text { connaissances tout le temps... construire quelque chose } \\
\text { de bien... utiliser de l'ancien avec du nouveau. » } \\
\text { (participant de Sudbury) }\end{array}$ \\
\hline & Microphone & $\begin{array}{l}\text { "On n'est pas assez à l'écoute... on parle trop : il faut } \\
\text { apprendre à savoir quels sont ses besoins [au client] } \\
\text { pour avoir un impact. Il faut qu'on les écoute. » } \\
\text { (participant de Toronto) }\end{array}$ \\
\hline \multirow[t]{2}{*}{$\begin{array}{l}\text { Changements } \\
\text { d'attitude }\end{array}$} & Pieuvre & $\begin{array}{l}\text { «L'aspect multidimensionnel... s'adapter à toutes sortes } \\
\text { de personnes... plusieurs bras, plus de chances de } \\
\text { rejoindre son auditoire. » (participant d'Ottawa) }\end{array}$ \\
\hline & $\begin{array}{l}\text { Soulier rose } \\
\text { de princesse }\end{array}$ & $\begin{array}{l}\text { "L'aspect culturel de la littératie en santé... la nécessité } \\
\text { d'adapter son message à la culture. » } \\
\text { (participant de Toronto) }\end{array}$ \\
\hline
\end{tabular}

Remarque : Ce tableau présente des exemples d'idées formulées par différents participants. L'information présentée n'est pas représentative d'un sens consensuel donné aux objets évocateurs.

a Il s'agit d'une traduction de la version anglaise approuvée par les auteurs.

les clients avaient aussi besoin d'une littératie technologique et informatique pour manipuler les appareils médicaux et les fournitures médicales électroniques.

Certains participants ont constaté que leurs clients francophones faisaient une utilisation stratégique de l'information en santé dans leur deuxième langue en se servant d'un jargon médical pour dissimuler leur incapacité à décoder l'information en santé rédigée en anglais. Leur utilisation du jargon médical les protègerait des professionnels qui s'interrogent sur leur capacité à comprendre l'information en santé. D'autres participants ont fait état des difficultés auxquelles font face les immigrants francophones en Ontario qui ne savent ni lire ni écrire en français. Ils peuvent aussi être incapables de se servir du réseau téléphonique pour obtenir des soins médicaux d'urgence ou pour avoir accès à des soins de santé primaires. Moins au courant que les non-immigrants du principe de prévention des maladies tel qu'il est appliqué au Canada, les immigrants sont susceptibles de moins bien comprendre les programmes de prévention des maladies. Les participants ont avancé l'idée que les problèmes de LS pourraient être atténués en privilégiant l'information sur la promotion de la santé axée sur les moyens de demeurer en bonne santé plutôt que l'information sur la prévention des maladies axée sur la probabilité de développer des maladies.

\section{Obstacles organisationnels à l'intégration de la littératie en santé dans la pratique}

$\mathrm{Au}$ cours du deuxième exercice, les participants ont raconté des histoires révélatrices à la fois des obstacles organisationnels, par exemple la courte durée de consultation avec les clients et l'emploi habituel du jargon médical dans le contexte professionnel, et des moyens d'application du concept de LS dans leur pratique. Les participants ont convenu que le langage des professionnels devait être adapté au milieu organisationnel et au niveau de littératie des clients et que les modes de communication eux-mêmes devaient être adaptés à la culture et au niveau de littératie des clients. Les participants ont considéré que le jargon médical constituait tout particulièrement un facteur nuisant à l'observance des traitements et à l'auto-prise en charge des maladies et empêchant les clients de décrire leurs symptômes simplement. Un participant a formulé le commentaire suivant :

...parce que si jamais un médecin ou n'importe quel professionnel de la santé, tu essaies d'expliquer à quelqu'un, comme elle disait, un concept que ça t'a pris 4 heures pour maitriser, et tu essaies de lui expliquer ça en 2 minutes en utilisant des termes vraiment techniques... (participant de Sudbury)

Les participants ont discuté de l'importance de parler aux clients dans leur langue maternelle. Par exemple, certains clients âgés, élevés en français et ayant étudié en français et en anglais, peuvent préférer parler en français et lire en anglais. Cette différence peut constituer un problème au sein des organismes qui offrent des documents d'information unilingues ou qui prétendent offrir des services bilingues mais ne communiquent verbalement qu'en anglais.

Mieux communiquer avec les clients a été considérée comme une stratégie de première importance pour que les efforts déployés par les professionnels, tant en clinique que sur le plan culturel, pour améliorer la LS de leurs clients portent leurs fruits. Certaines techniques de communication (p. ex. parler lentement, répéter l'information, utiliser des phrases plus simples) ont été jugées cruciales pour améliorer les connaissances en santé des clients incapables d'avoir accès à des services en français.

Les participants ont considéré également que la structure organisationnelle et la culture au sein du système de santé, en particulier le manque de temps et de souplesse, limitaient l'intégration de la LS à leur pratique professionnelle. Le temps manque souvent pour expliquer les con- 
cepts et pour s'attacher aux indices non verbaux des clients et aux contextes sociaux complexes. Un participant de Sudbury a expliqué : « Nous jugeons qu'il est très très important de bien écouter le langage - tant verbal que non verbal - qu'utilisent les gens, de s'attarder aux petits signes, à la façon dont ils parlent. Nous devrions aussi trouver des moyens pour mieux connaître notre clientèle. ${ }^{\dagger}$ Un autre participant (d’Ottawa) a suggéré d'atténuer les contraintes de temps en élaborant des plans avec les clients et d'autres professionnels pour changer les approches en ce qui concerne l'information, les soins et la prestation des services en santé : " Nous devons prévoir, planifier et prendre du temps avec nos clients parce que nous avons dit que nous sommes souvent limités. [Par exemple,] si vous allez voir votre médecin de famille, vous n'avez que huit minutes. $»^{\dagger}$

Si les participants ont réfléchi aux moyens d'intégrer le concept de LS au niveau individuel et au niveau collectif, ils ont perçu moins d'occasions de le faire au niveau organisationnel, peut-être à cause des contraintes budgétaires et des priorités des décideurs. Les formulaires de rétroaction ont indiqué un gain dans trois domaines de conscientisation au sujet de la LS : en tant que concept, en tant que déterminant social de la santé et en tant qu'outil individuel, collectif et organisationnel pour améliorer la santé. De nombreux participants ont souhaité la tenue d'autres ateliers et davantage d'occasions d'échange des connaissances avec d'autres professionnels, de façon à élargir leurs connaissances et leurs compétences en matière de LS. Certains ont demandé une formation continue pour apprendre à mieux tenir compte de la diversité culturelle francophone lors de la prestation de services de santé ou de services sociaux :

Nous avons besoin d'ateliers portant sur les compétences culturelles. C'est un gros problème pour les gens de notre communauté, parce que la communauté francophone est très vaste. Les gens arrivent de partout, mais ils arrivent avec des différences culturelles. (participant de Toronto) $)^{\dagger}$

\section{Analyse des résultats de l'étude d'évaluation}

Les idées communes de Freire $^{29}$ et d'Ekebergh ${ }^{47}$ au sujet de l'apprentissage de son monde et de la place qu'on occupe dans le monde au moyen d'une réflexion individuelle consciente ont servi de fondement à l'analyse et à l'interprétation des données d'évaluation de l'atelier. Tant Freire qu'Ekebergh affirment qu'en prenant du recul par rapport à nos expériences quotidiennes et en réfléchissant de façon critique sur notre propre réalité, nous augmentons notre conscience critique. Les participants à l'atelier ont réfléchi à propos de leur connaissance expérientielle du combat que mènent les Canadiens francophones pour avoir accès à de l'information et à des services de santé en français, et au sujet de la façon dont la LS influe sur ces deux éléments. Les objets évocateurs que l'animatrice a remis aux participants et qui ont suscité des discussions au sujet des expériences personnelles et professionnelles ont été à l'origine de cette réflexion. Les participants ont discuté de la façon dont le mode de communication de l'information en santé avec les clients influe sur la compréhension que ces derniers ont de cette information, ainsi que sur la manière dont les clients peuvent l'appliquer pour se soigner et prendre en charge eux-mêmes leurs maladies. Notre analyse des données d'évaluation de l'atelier ont révélé trois phénomènes chez les participants : un élargissement de la vision à propos de la LS, des changements d'attitude au sujet de la LS et une réflexion sur les possibilités de modification des pratiques professionnelles.

\section{Élargiissement de la vision des participants à propos de la LS}

Les participants à l'atelier ont accordé la même valeur à leurs connaissances expérientielles et à leurs nouvelles connaissances. Ils ont aussi partagé leur compréhension des relations de pouvoir entre professionnels et entre professionnels et clients. Ils ont défini leur vision de la LS, l'élargissant de ses dimensions physique et mentale à ses dimensions sociale et politique. Ils ont commencé à ressentir de l'empathie envers leurs clients à faible littératie en santé et les expériences que ceux-ci vivent, et ils ont pris conscience de la stigmatisation associée à une piètre compréhension de l'information en santé et de la difficulté à prendre des décisions en santé sans comprendre le jargon médical ou les options thérapeutiques et leurs conséquences. De plus, les clients ont souvent des réactions émotives et psychosociales lorsqu'on utilise le jargon médical pour leur fournir de l'information, ce qui peut les empêcher de comprendre et de respecter les directives et nuire à la prise des décisions ${ }^{48}$. L'échange d'idées et d'expériences a permis aux participants de prendre conscience de la façon dont la LS et d'autres déterminants sociaux de la santé se conjuguent et comment cette conjugaison influe sur l'accès aux services de santé et aux services sociaux ${ }^{48}$. Cette vision plus large de la LS, qui découle d'un décodage du monde et de réflexions sur ce monde, peut aider à promouvoir encore davantage la $\mathrm{LS}^{49,50}$ :

Cet atelier m'a permis de comprendre le concept, la notion de littératie en santé et m’a amené à la relier aux caractéristiques sociales... [J'ai compris] que la littératie englobe aussi le bagage culturel, les constructions sociales liées à la maladie et au système de santé. (participant d'Ottawa) ${ }^{\dagger}$

\section{Changements d'attitude des participants à propos de la LS}

Les discussions de groupe ont révélé un changement d'attitude des participants au sujet de la LS au cours de l'atelier. Les participants ont pris conscience du fait que la faible LS parmi leurs clients francophones était en fait un problème plus général qui allait au-delà du manque de services en français et que d'importants problèmes de droit, d'éthique et de gestion devaient être pris en compte et abordés dans toutes les institutions de santé et de services sociaux qui desservent cette clientèle. Les participants ont affirmé leur intention de défendre le droit de leurs clients à des services et à de l'information en santé en français et de repérer les

† Il s'agit d'une traduction de la version anglaise approuvée par les auteurs. 
iniquités basées sur la langue dans les services. Ces intentions ont découlé d'une meilleure compréhension des difficultés auxquelles font face les clients à faible littératie en santé à tous les niveaux de soins. En identifiant les obstacles à la prise de décision rencontrés par leurs clients (que ce soit le manque d'information, l'incapacité à prévoir ou l'incapacité à comprendre les risques), les participants ont pris conscience de leur part de responsabilité dans l'éducation et le soutien des clients quant à la prévention et la prise en charge des maladies, car ils ont pris conscience du rôle de la LS dans la capacité de compréhension de leurs clients :

Je comprends que les enjeux sont plus étendus que je ne le croyais, qu'ils vont bien au-delà de la lecture, qu'ils constituent un danger pour la santé des individus. (participant de Toronto)

La réflexion individuelle a provoqué une plus grande conscientisation (voir les tableaux 3 et 4) du droit des clients à des services et à de l'information en santé en français dans le milieu de travail des participants et ailleurs. En vivant des difficultés à se remémorer les connaissances professionnelles que suscitaient les objets évocateurs, les participants se sont rendu compte à quel point ces objets simples et ludiques provoquaient une conscientisation. Les objets ont donné naissance à des analogies et à des métaphores riches de sens. Ayant pris davantage conscience des difficultés de communication, les participants ont énuméré, comme facteurs rendant plus difficiles les soins axés sur le client, les contraintes de temps, les budgets limités, le jargon professionnel, un matériel écrit complexe et de fortes demandes de services. Le manque de temps pour bien expliquer les concepts et les schémas thérapeutiques a été considéré comme le principal obstacle à une communication efficace en santé. Cette meilleure prise de conscience des difficultés en LS qu'éprouvent les minorités linguistiques en lien avec la LS a incité les participants à modifier leur pratique en utilisant un langage simple et en consacrant plus de temps à l'écoute de leurs clients.

\section{Réflexion au sujet de la possibilité de} modification par les participants de leurs pratiques professionnelles

L’atelier a donné aux participants l'occasion de faire une séance de remueméninges concernant les outils, les compétences et les stratégies pouvant servir à promouvoir la LS dans leur pratique professionnelle. La diversité des disciplines et des cultures des participants a permis de renforcer les stratégies suggérées et d'accroître leur validité, car nombre d'entre elles reposaient sur l'expérience. La mise au point de stratégies et l'identification d'outils ont fait ressortir à quel point les participants maîtrisaient les nouvelles ressources à leur disposition pour promouvoir la LS et en apprendre davantage à son sujet. Par exemple, certains organismes communautaires se servent maintenant de
Twitter pour promouvoir la LS et faire mieux comprendre à leurs clients l'information de base en santé. De plus, des organismes gouvernementaux accordent plus d'attention à la diversité culturelle dans leurs messages sur Twitter ${ }^{51}$.

Les participants ont pu tabler sur leurs expériences en tant que professionnels francophones pour évaluer de façon critique les données de recherche concernant la LS et les traduire d'une façon adaptée à leur pratique. Ils ont utilisé les connaissances acquises au sujet de la LS afin de suggérer de nouvelles stratégies pour améliorer les services (voir le tableau 5). Des données scientifiques ciblées devraient toujours être utilisées lorsqu'on met sur pied des interventions innovatrices à l'intention des personnes à faible littératie en santé ${ }^{52}$. Une attention particulière devrait être portée aux moyens de communication préférés des clients (p. ex. romans-photo, vidéos). Une approche axée sur les préférences culturelles des clients pour la communication en santé s'est par exemple avérée efficace pour entrer en contact avec les immigrants canadiens souffrant d'asthme ${ }^{53}$.

Le milieu organisationnel décrit par les participants semble peu favorable en tant que tel aux interventions visant à améliorer la LS. Par conséquent, les changements dans la pratique professionnelle nécessitent un soutien organisationnel accru par l'entremise d'un plus grand accès à des ressources matérielles et professionnelles, ce que recommandent

TABLEAU 4

Opinions des participants à l'atelier « Placez la littératie en santé au cœur de la pratique » sur la littératie en santé

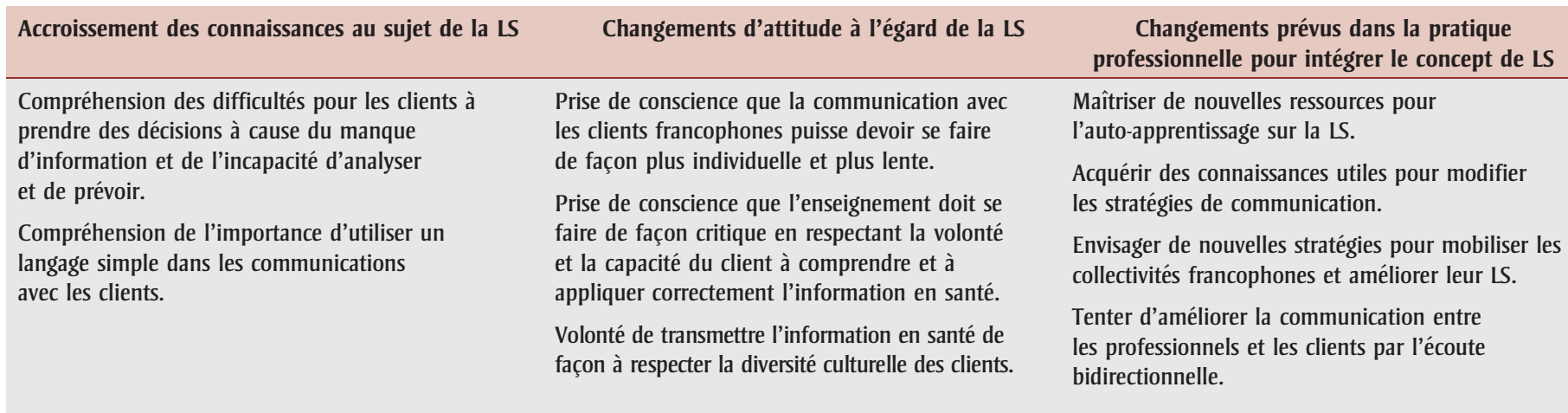

Abréviation : LS, littératie en santé.

Il s'agit d'une traduction de la version anglaise approuvée par les auteurs. 
TABLEAU 5

Recommandations pour intégrer le concept de littératie en santé à la pratique

\begin{tabular}{|c|c|}
\hline Population cible & Recommandations des participants à l'atelier ${ }^{\mathrm{a}}$ et des auteurs \\
\hline \multirow[t]{3}{*}{ Professionnels } & Faire connaître à la clientèle le concept de LS. \\
\hline & $\begin{array}{l}\text { Établir des réseaux pour encourager le dialogue entre les praticiens communautaires } \\
\text { au sujet de la LS. }\end{array}$ \\
\hline & $\begin{array}{l}\text { Communiquer l'information à d'autres pour faire connaître à la collectivité les } \\
\text { ressources disponibles et les interventions possibles en matière de LS. }\end{array}$ \\
\hline Gestionnaires & $\begin{array}{l}\text { Favoriser les changements organisationnels afin d'aider les professionnels à } \\
\text { s'adapter aux nouvelles réalités sociales de leurs clients et à reconnaître les } \\
\text { besoins en matière de LS à l'échelle de l'individu, de la communauté } \\
\text { et de l'organisation. Créer une vision organisationnelle afin de régler les } \\
\text { problèmes de LS dans les minorités linguistiques. }\end{array}$ \\
\hline \multirow[t]{5}{*}{ Chercheurs } & $\begin{array}{l}\text { Concevoir des méthodes innovatrices pour étudier les problèmes sociaux émergents } \\
\text { liés à la LS en tablant sur l'expertise de professionnels en contact direct } \\
\text { avec la clientèle. }\end{array}$ \\
\hline & $\begin{array}{l}\text { Déterminer les obstacles organisationnels à l'intégration du concept de LS dans la } \\
\text { pratique professionnelle. }\end{array}$ \\
\hline & $\begin{array}{l}\text { Étudier des moyens de faire participer les clients à la prise de décisions et aux } \\
\text { changements organisationnels. }\end{array}$ \\
\hline & $\begin{array}{l}\text { Concevoir des recherches participatives qui font appel tant à ceux qui organisent } \\
\text { et donnent les soins de santé et les services sociaux qu'aux clients dans des } \\
\text { communautés francophones minoritaires. }\end{array}$ \\
\hline & Évaluer les répercussions à long terme d'ateliers similaires. \\
\hline \multirow[t]{5}{*}{ Éducateurs } & $\begin{array}{l}\text { Concevoir des stratégies pour démystifier les résumés de recherche, les concepts } \\
\text { complexes pour les professionnels. }\end{array}$ \\
\hline & Créer des outils de communication pour faire le lien entre la théorie et la pratique. \\
\hline & $\begin{array}{l}\text { Aider les professionnels, par l'apprentissage participatif, à mieux comprendre la } \\
\text { théorie qui sous-tend la pratique. }\end{array}$ \\
\hline & $\begin{array}{l}\text { Promouvoir les activités faisant mieux connaître les éléments qui nuisent à } \\
\text { l'accès à l'information en matière de santé et de services sociaux à l'échelle } \\
\text { du client, du professionnel, de l'organisation et du système. }\end{array}$ \\
\hline & $\begin{array}{l}\text { Offrir des ateliers similaires à des étudiants francophones en sciences de la santé } \\
\text { et en sciences sociales de niveau post-secondaire afin qu'ils puissent plaider en } \\
\text { faveur d'un meilleur accès aux services pour les minorités linguistiques francophones }\end{array}$ \\
\hline
\end{tabular}

Abréviation : LS, littératie en santé.

a «Placez la littératie en santé au cour de la pratique ».

DeWalt et collab. ${ }^{54}$. Les organismes qui offrent des soins de santé primaires devraient adopter des programmes d'amélioration de la qualité pour mettre en œuvre les interventions visant à améliorer la LS, « [...] promouvoir les services en français et promouvoir la littératie en santé, donc développer cette sensibilité et cette compétence culturelle, libérer le personnel pour qu'il puisse suivre des formations, se centrer sur le client et cerner ses besoins. » (participant d'Ottawa) ${ }^{\S}$

Les changements annoncés dans la pratique se sont exprimés sous forme de plans d'action parce que les participants se sont rendu compte qu'ils possédaient le savoir expérientiel requis pour promouvoir et susciter ces changements. En maîtrisant le processus d'apprentissage qu'ils ont vécu, les participants peuvent être en mesure de le reproduire avec leur clientèle. Leur expérience de décodage des sens potentiels de chaque objet évocateur les a placés dans une position comparable à celle de leurs clients qui tentent de décoder l'information en santé, les conscientisant par conséquent aux épreuves auxquelles les clients sont confrontés. Ce décodage, aspect primordial de la LS, peut influencer la manière dont les participants vont modifier leur mode d'enseignement aux clients en ce qui concerne la préven- tion et la prise en charge des blessures et des maladies chroniques. Cet atelier a permis de faire prendre conscience aux participants de l'importance de la LS pour les francophones en situation de minorité linguistique et les a incités à vouloir modifier leur pratique professionnelle (voir le tableau 5) et à militer en faveur de changements organisationnels.

\section{Analyse}

Notre recension des écrits ne nous a pas permis de relever d'étude ayant fait appel à des objets évocateurs pour susciter chez les professionnels une réflexion au sujet de l'univers culturel et des expériences de vie des apprenants ${ }^{31}$. La plupart des études ayant porté sur les méthodes d'apprentissage participatif pour la formation des professionnels de la santé ne donnent aucun détail sur les théories et les philosophies pédagogiques qui sous-tendent ces méthodes. Il est donc difficile de comparer nos résultats à ceux d'autres études. Nous avons tout de même relevé dans les écrits certaines stratégies d'apprentissage comme les discussions, les interactions en groupe et les jeux de rôle ${ }^{55}$. Certaines études font également état d'une formation multidisciplinaire en services communautaires visant à susciter la communication et la collaboration entre professionnels parmi les étudiants en sciences de la santé et en services sociaux $^{56}$. Même si l'on est conscient de l'importance des compétences linguistiques des clients pour que ceux-ci puissent bénéficier pleinement des soins de santé et des services sociaux, cette question n'a pas été véritablement examinée dans le contexte de la formation professionnelle. On peut citer cependant une étude menée par Sullivan et collab. ${ }^{30}$ au moyen d'une série de quatre ateliers visant à augmenter la capacité des professionnels à communiquer avec une clientèle multilingue, étude dans laquelle les discussions de groupe constituaient la principale méthode employée. Dans une autre étude, on a appliqué les principes éducatifs de Freire au moyen de théâtre, d'histoires et de photos $^{57}$ dans le cadre d'une formation des travailleurs en santé communautaire sur l'organisation et de la mobilisation

$\S$ Il s'agit d'une traduction de la version anglaise approuvée par les auteurs. 
communautaires $^{58}$. Globalement, le manque d'information sur la conception de l'atelier dans plusieurs études ne permet pas d'analyser plus avant la pertinence d'autres stratégies de formation continue.

Les résultats de notre étude d'évaluation corroborent les écrits existants sur les obstacles généraux que doivent affronter les professionnels francophones qui tentent d'améliorer les conditions sanitaires et sociales des populations francophones en situation minoritaire ${ }^{59}$. En bref, peu d'organismes se sont donné des mandats ou des mécanismes officiels pour contrer directement les problèmes de LS au sein de leurs services ${ }^{60}$. Pour la plupart des organismes de santé et de services sociaux, la prestation de services (si nécessaire) aux francophones incombe aux intervenants et décideurs anglophones $^{61}$. Les professionnels de la santé présument sans doute que leurs clients comprennent leurs conseils parce que peu de clients demandent des services en français ${ }^{61}$. Cependant, mieux former les clients en LS pour éliminer les obstacles à la communication ${ }^{62}$ est insuffisant si les professionnels eux-mêmes ne sont pas formés $^{63}$ à la promotion de la LS chez les minorités linguistiques.

Les participants à l'atelier ont cité plusieurs stratégies pour améliorer la façon dont ils voudraient diffuser l'information en santé. En premier lieu, ils ont suggéré de diversifier l'approche pédagogique en utilisant du matériel audiovisuel, en particulier pour les personnes âgées. En deuxième lieu, ils ont souligné l'importance d'établir des relations plus fortes avec leurs clients et d'améliorer la communication orale. En troisième lieu, ils ont considéré la LS comme relevant de la santé publique parce qu'elle a une incidence sur la capacité des clients à comprendre les risques, à prendre en charge leurs problèmes de santé et à adopter des comportements bénéfiques en matière de prévention et de dépistage. Ce point de vue corrobore celui relevé dans d'autres travaux de recherche selon lequel la LS et la littératie générale, dont la capacité à comprendre les risques et les probabilités, doivent être incluses dans les mesures de santé publique ${ }^{7}$. Les participants ont souligné l'importance de la communica- tion en langage simple, ce qui est une pratique exemplaire recommandée ${ }^{40,62,64}$. Bien que la majorité des participants aient reconnu qu'améliorer les communications et le matériel éducatif était une nécessité, ils se sont rendu compte que la collaboration avec les clients pour trouver des solutions était impossible à cause des contraintes de leur milieu de travail et de leurs activités.

En quatrième lieu, les participants ont suggéré d'utiliser la formation continue pour aider les professionnels à mieux tenir compte de la LS dans leur pratique. Plus leurs compétences dans ce domaine augmenteront, plus les professionnels seront portés à vérifier que leurs clients comprennent l'information en santé et pourront mieux adapter le matériel éducatif aux besoins des clients à faible littératie ${ }^{65}$.

En cinquième et dernier lieu, les participants ont appris que la LS agissait en synergie avec la langue (dont les accents) et la culture (notamment les croyances en matière de santé, l'âge, le statut à l'égard de l'immigration, le soutien social, le niveau de scolarité et le statut socioéconomique). Ils ont donc jugé nécessaire une formation continue sur les compétences culturelles et la prestation de services adaptés sur le plan culturel qui inciterait les professionnels à concevoir, donner et évaluer ce type de services ${ }^{66}$. Ils ont jugé tout aussi nécessaire une formation en matière de communication interculturelle en santé pour les éducateurs en santé et les consommateurs ${ }^{67}$. Cependant, ce qui confirme les résultats de Herndon et collab. ${ }^{24}$, les participants ont reconnu que le manque de temps constituait un obstacle organisationnel majeur à la promotion de la LS.

Il faut poursuivre la conscientisation à l'égard de la LS si l'on veut que les professionnels puissent améliorer la LS de leurs clients ${ }^{63}$. Tous les professionnels de la santé, y compris les médecins et les étudiants en médecine, ont besoin de davantage de formation en $\mathrm{LS}^{63}$. Les infirmières et les étudiants en sciences infirmières, qui se chargent habituellement de l'enseignement aux patients mais qui sont moins susceptibles que les médecins d'évaluer la LS des patients ${ }^{68}$, doivent connaître des méthodes simples d'évaluation de la LS dans le cadre des soins primaires ${ }^{19,69}$. Étant donné que la LS des clients pourra difficilement s'améliorer si l'on continue à utiliser de l'information en santé imprimée dans des langues dont ils ne maîtrisent pas la lecture, des formats non imprimés devraient être envisagés pour les clients à faible littératie ${ }^{55,70}$.

\section{Limites de l'étude}

Notre étude d'évaluation comporte plusieurs limites. Premièrement, ce sont peutêtre l'obligation pour les organismes d'offrir des services bilingues et les objectifs de perfectionnement professionnel établis par les participants à l'atelier eux-mêmes qui ont incité ces derniers à participer à l'atelier. Deuxièmement, nous avons analysé uniquement le point de vue des dispensateurs de services et non celui des clients ou des directeurs d'organismes de santé et de services sociaux. Troisièmement, les répercussions de l'atelier ont été recueillies par le biais de formulaires de rétroaction remplis tout de suite après l'atelier, ce qui est une méthode vulnérable aux réactions enthousiastes susceptibles d'être tempérées ensuite après réflexion sur l'utilité du travail.

Notre étude comporte aussi plusieurs biais potentiels. Premièrement, les participants se considéraient eux-mêmes comme professionnels francophones travaillant avec des Franco-Ontariens, ce qui peut les avoir sensibilisé davantage aux problèmes de LS. Deuxièmement, les participants travaillaient tous pour des organismes ayant pour mandat d'offrir des services en français, ce qui a pu susciter un sentiment d'obligation professionnelle d'être au fait des problèmes de LS que vivent leurs clients francophones. Troisièmement, les chercheurs qui ont travaillé sur les transcriptions n'étaient pas de langue maternelle française, ce qui a pu se solder par des interprétations erronées des propos des participants. Pour contrer ce problème potentiel, ce sont des francophones de langue maternelle qui ont transcrit les enregistrements et des chercheurs francophones ont participé à l'atelier et à l'analyse des données. Un travailleur francophone a confirmé l'interprétation finale des résultats. 


\section{Conclusion}

Les participants à nos ateliers ont estimé que la prise de conscience des problèmes de LS relevait d'une responsabilité collective. Le fait que les participants déclarent qu'ils avaient tous la responsabilité de modifier leur milieu de travail et leur pratique individuelle et collective confirme la justesse de la théorie de la pédagogie critique de Freire $^{29}$ utilisée pour l'atelier. Cette responsabilisation collective corrobore aussi notre conviction que la pédagogie critique appliquée à la formation continue suscite véritablement chez les professionnels une prise de conscience de leur capacité à modifier leur pratique et leur milieu de travail et à participer au changement social. Les programmes de perfectionnement professionnel devraient utiliser l'apprentissage critique et offrir des outils efficaces pour faire face à la complexité grandissante de la LS dans des sociétés multiculturelles et multilingues. Pour être efficaces, ces outils devraient avoir un sens pour les apprenants professionnels. Freire conseille d'utiliser de manière générale des outils d'apprentissage ayant un sens pour les apprenants ${ }^{71}$. De plus, pour lui, il existe une activité fondamentalement formatrice pour les éducateurs : la réflexion critique sur leur pratique et leurs conceptions de l'avenir ${ }^{71}$. Cette réflexion devrait porter sur les rêves, sur les idées novatrices et sur les objectifs des éducateurs, éléments qui révèlent tous les racines politiques des activités éducatives. Durant le processus de promotion de la LS, les professionnels francophones et leurs clients partagent une même réalité sociale : la vie en situation de minorité linguistique. Toutefois, les professionnels n'étaient pas forcément conscients de la dimension politique de ce statut de minorité dans leur propre vie. Notre utilisation de la théorie de la pédagogie critique de Freire s'est révélée de ce fait adéquate pour promouvoir l'autonomie des apprenants quant à la critique de leur réalité sociale. C'est précisément le statut de minorité linguistique des participants qui a guidé le choix de notre approche philosophique plutôt que de celle de présentation de données empiriques, issues la plupart du temps d'études menées auprès de populations aux réalités culturelles et linguistiques différentes.
Nos résultats pourraient s'appliquer à d'autres minorités linguistiques canadiennes et aux organismes qui élaborent des politiques en matière de santé et de services sociaux pour ces minorités, ainsi qu'aux pays qui ne disposent pas de politiques relatives à une deuxième langue ou à plusieurs langues.

Les professionnels de la santé et des services sociaux de l'Ontario ont acquis une expertise de travail dans des contextes multiculturels. En conséquence, nos résultats pourraient être utiles dans des milieux de pratique similaires et favoriseraient la compréhension de la manière dont la langue, l'accès aux soins de santé et aux services sociaux et d'autres déterminants sociaux de la santé influent sur la santé des minorités linguistiques, y compris des immigrants $^{72}$. Nos résultats pourraient être également utiles lors de la conception de stratégies éducatives en santé pour les groupes immigrants qui ont une piètre connaissance du français et de l'anglais. De même, l'utilisation d'objets évocateurs pourrait être une méthode d'apprentissage intéressante pour d'autres groupes, tels ceux à risque de blessures (p. ex. les jeunes qui pratiquent des sports saisonniers) et pour stimuler les professionnels à concevoir des moyens adaptés de promotion de la santé pour les populations aux prises avec des maladies chroniques.

\section{Remerciements}

Nous remercions les professionnels et les étudiants dans le domaine de la santé et des services sociaux qui ont participé à l'atelier. Nous tenons également à remercier les $\mathrm{D}^{\text {res }}$ Manon Lemonde et Joëlle Fareau-Weyl pour leur relecture de la première ébauche de ce manuscrit ainsi que Mme Margaret Oldfield et la $\mathrm{D}^{\mathrm{re}}$ Sylvia Novac pour leur révision du manuscrit.

Nous n'avons aucun conflit d'intérêts à déclarer.

Notre étude a été rendue possible grâce au soutien financier du Conseil canadien sur l'apprentissage, à une subvention incitative de l'Université York, à une subvention pour initiative spéciale au premier cycle de l’Université Ryerson et à la Writing Week
Initiative de la Faculté des services communautaires de l'Université Ryerson.

\section{Références}

1. Barrett SE, Puryear JS. Health literacy: improving quality of care in primary care settings. J Health Care Poor Underserved. 2006;17(4):690-697.

2. Gazmararian JA, Williams MV, Peel J, Baker DW. Health literacy and knowledge of chronic disease. Patient Educ Couns. 2003;51:267-275.

3. Cronin M, Connolly C. Exploring the use of experiential learning workshops and reflective practice within professional practice development for post-graduate health promotion students. Health Educ J. 2007;66(3):286303.

4. de Barros D, Barbieri AR, Ivo ML et collab. O contexto da formação dos agentes comunitários de saúde no Brasil. [Contexte de formation des agents de santé communautaire au Brésil]. Texto \& Contexto Enfermagem. 2010;19(1):78-84. (en portugais)

5. Chávez V, Turalba RA, Malik S. Teaching public health through a pedagogy of collegiality. Am J Public Health. 2006;96(7):11751180.

6. Rootman I, Gordon-El-Bihbety D, Frankish J et collab. Programme national de recherches sur l'alphabétisation et la santé : évaluation des besoins et analyse du contexte. Ottawa (Ont.) : Association canadienne de santé publique; 2003.

7. Kaszap M, Zanchetta MS. La littératie en santé, vécue dans la simplicité mais comprise à travers la complexité : regard sur les communautés culturelles (francophones, minoritaires et multiethniques). Dans: Masny D, dir. Les littératies multiples : lire au $21^{\mathrm{e}}$ siècle. Ottawa : Presses de l'Université d'Ottawa; 2009. p. 287-325.

8. Forgues É, Landry R. Définitions de la francophonie en situation minoritaire : analyse de différentes définitions statistiques et de leurs conséquences. Ottawa (Ont.) : Consortium national de formation en santé; 2006. 
9. Bouchard L, Gaboury I, Chomienne M-H, Gilbert A, Dubois L. Health in language minority situation. Healthc Policy. 2009;4: 36-42.

10. Deveau K, Landry R, Allard R. Au-delà de l'autodéfinition. Composantes distinctes de l'identité ethnolinguistique. Francophonies d’Amérique. 2005;20:79-93.

11. Office des affaires francophones. Profil de la communauté francophone de l'Ontario 2009. Toronto (Ont.) : Office des affaires francophones, La fondation Trillium de l'Ontario; 2009. PDF (2,65 Mo) téléchargeable à partir du lien : http://docs.files.ontario.ca/documents /410/profil-des-francophones.pdf

12. Corbeil J, Lafrenière S. Portrait des minorités de langue officielle au Canada : les francophones de l'Ontario. Ottawa (Ont.) : Statistique Canada; 2010. (n 89-642-X au catalogue)

13. Conseil canadien sur l'apprentissage. La littératie en santé au Canada : une question de bien-être [Internet]. Ottawa (Ont.) : Conseil canadien sur l'apprentissage; 2008 [consulté le 29 nov. 2009]. Consultable en ligne à partir de la page : http://www.ccl-cca .ca/CCL/Reports/HealthLiteracy-2.html

14. Rootman I, El-Bihbety D. Vision d'une culture de la santé au Canada : Rapport du Groupe d'experts sur la littératie en matière de santé. Ottawa (Ont.) : Association canadienne de santé publique; 2008.

15. Papen U. Adult literacy as social practice : more than skills. New York (NY) : Routledge; 2005.

16. Schonlau M, Martin L, Haas A, Derose KP, Rudd R. Patients' literacy skills : more than just reading ability. J Health Commun. 2011;16(10):1046-1054.

17. St. Clair R. Why literacy matters - understanding the effects of literacy education for adults. Leicester (RU) : National Institute of Adult Continuing Education; 2010.

18. Bouchard L, Desmeules M. Minorités de langue officielle du Canada. Québec (Qc) : Presses de l’Université du Québec; 2011.

19. Berkman ND, Davis TC, McCormack L. Health literacy: what is it? J Health Commun. 2010;15(Suppl.2):9-19.
20. Howard DH, Sentell T, Gazmararian JA. Impact of health literacy on socioeconomic and racial differences in health in an elderly population. J Gen Intern Med. 2006;21(8): 857-861.

21. Zanchetta MS, Poureslami IM. Littératie en matière de santé dans la réalité des immigrants, sur le plan de la culture et de la langue. Revue canadienne de santé publique. 2006;97(Suppl.2):S28-33.

22. Conseil canadien sur l'apprentissage. État de l'apprentissage au Canada : Pas le temps de s'illusionner. Ottawa (Ont.) : Conseil canadien sur l'apprentissage; 2007.

23. Baker DW. The meaning and the measure of health literacy. J Gen Intern Med. 2006;21(8):878-83.

24. Herndon JB, Chaney M, Carden D. Health literacy and emergency department outcomes: a systematic review. Ann Emerg Med. 2011;57(4):334-345.

25. Perlow E. Accessibility: global gateway to health literacy. Health Promot Pract. 2010;11(1):123-131.

26. Paasche-Orlow MK, Schillinger D, Greene SM, Wagner EH. How health care systems can begin to address the challenge of limited literacy. J Gen Intern Med. 2006; 21(8):884-887.

27. Volandes AE, Paasche-Orlow MK. Health literacy, health inequality and a just healthcare system. Am J Bioeth. 2007;7(11): 5-10.

28. Rudd RE, Rosenfeld L, Simonds VW. Health literacy: a new area of research with links to communication. Atl J Commun. 2012;20(1): 16-30.

29. Freire P. Education for critical consciousness. New York (NY) : Continuum; 1973.

30. Sullivan MF, Ferguson W, Haley HL et collab. Expert communication training for providers in community health centers. J Health Care Poor Underserved. 2011;22(4):1358-1368.

31. Wallerstein N, Bernstein E. Empowerment education: Freire's ideas adapted to health education. Health Educ Q. 1988;5:379-394.
32. Wallerstein N. Powerlessness, empowerment, and health: implications for health promotion programs. Am J Health Promotion. 1992;6:197-205.

33. Freire P. Pedagogy of hope: reliving the pedagogy of the oppressed. New York (NY) : Continuum; 2003.

34. Freire P. Educação como prática da liberdade [Éducation comme pratique de la liberté]. $23^{\mathrm{e}}$ éd. Rio de Janeiro (BR) : Paz e Terra; 1999. (en portugais)

35. Freire P. Educação e mudança [Éducation et changement]. $23^{\mathrm{e}}$ éd. Rio de Janeiro : Paz e Terra; 1999. (en portugais)

36. Freire P. Ação cultural para a liberdade e outros escritos [Action culturelle pour le changement et autres écrits]. $10^{\mathrm{e}}$ éd. São Paulo : Paz e Terra; 2002. (en portugais)

37. Racine L. Applying Antonio Gramsci's philosophy to postcolonial feminist social and political activism in nursing. Nurs Philos. 2009;10(3):180-190.

38. Schwandt TA. Constructivist, interpretivist approaches to human inquiry. Dans : Denzin NK, Lincoln YS, dir. Handbook of qualitative research. Thousand Oaks : Sage; 1994. p. 118-37.

39. Tobin K, Tippins D. Constructivism as a referent for teaching and learning. Dans: Tobin K, dir. The practice of constructivism in science education. Hillsdale : Lawrence Erlbaum; 1993. p. 3-22.

40. Conseil canadien sur l'apprentissage. La littératie en santé au Canada : Une question de bien-être. Ottawa (Ont.) : Conseil canadien sur l'apprentissage; 2008.

41. Picard L, Allaire G. Deuxième rapport sur la santé des francophones de l'Ontario. Sudbury (Ont.) : Institut franco-ontarien, Université Laurentienne; 2005. PDF $(2,8$ Mo) téléchargeable à partir du lien : http:// www.sdhu.com/uploads/content/listings /rapport_sante_jan06.pdf

42. Freire P. Conscientização. Teoria e prática da libertação - Uma introdução ao pensamento de Paulo Freire [Conscientisation. Théorie et pratique de la libération. Introduction à la pensée de Paulo Freire]. São Paulo (BR) : Centauro; 2001. (en portugais) 
43. Rossi PH, Lipsey MW, Freeman HE. Evaluation: a systematic approach. $7^{\mathrm{e}}$ éd. Thousand Oaks : Sage; 2004.

44. Bardin L. L'analyse de contenu. $2^{\mathrm{e}}$ éd. Paris : Presses Universitaires de France; 2007.

45. Sandelowski M. The call to experts in qualitative research. Res Nurs Health. 1998;21(5):467-471.

46. Conseil canadien sur l'apprentissage. La littératie en santé au Canada [carte interactive de la littératie en santé au Canada]. Ontario [Internet]. Ottawa (Ont.) : Conseil canadien sur l'apprentissage; 2003 [consulté le 10 oct. 2008]. Consultable en ligne à partir de la page : http://www.ccl-cca .ca/cclflash/healthliteracy/map_canada_f .html

47. Ekebergh M. Lifeworld-based reflection and learning: a contribution to the reflective practice in nursing and nursing education. Reflective Practice. 2007;8(3):331-343.

48. Roter DL. Oral literacy demand of health care communication: challenges and solutions. Nurs Outlook. 2011;59(2):79-84.

49. Zanchetta M, Kaszap M, Mohamed M et collab. Construction of Francophone families' health literacy in a linguisticminority situation. Alterstice. 2012;2(2):4762. (Synthèse en français téléchargeable à partir du lien : http://journal.psy.ulaval.ca/ojs /index.php/ARIRI/article/view/Zanchetta \% 5BFR \% 5D_Alterstice2 \% 282\%29/pdf)

50. Wink J. Critical pedagogy : notes from the real world. $4^{\mathrm{e}}$ éd. Saddle River; 2011.

51. Park H, Rodgers S, Stemmie J. Analyzing health organizations' use of Twitter for promoting health literacy. J Health Commun. 2013;18(4):410-425.

52. Sheridan SL, Halpern DJ, Viera AJ, Berkman ND, Donahue KE, Crotty K. Interventions for individuals with low health literacy: a systematic review. J Health Commun. 2011;16(Suppl.3):30-54.

53. Poureslami I, Rootman I, Doyle-Waters MM, Nimmon L, Fitzgerald JM. Health literacy, language, and ethnicity-related factors in newcomer asthma patients to Canada: a qualitative study. J Immigr Minor Health. 2011;13(2):315-322.
54. DeWalt DA, Broucksou KA, Hawk V et collab. Developing and testing the health literacy universal precautions toolkit. Nurs Outlook. 2011;59(2):85-94.

55. Hershberger PJ, Righter EL, Zryd TW, Little DR, Whitecar PS. Implementation of a process-oriented cultural proficiency curriculum. J Health Care Poor Underserved. 2008;19(2):478-483.

56. Nandiwada DR, Dang-Vu C. Transdisciplinary health care education : training team players. J Health Care Poor Underserved. 2010;21(1): 26-34.

57. Porr CJ, Mayan M, Graffigna G, Wall S, Vieira, ER. The evocative power of projective techniques for the elicitation of meaning. Int J Qual Meth. 2011;10(1):30-41.

58. Perez M, Findley SE, Mejia M, Martinez J. The impact of community health worker training and programs in NYC. J Health Care Poor Underserved. 2006;Suppl:26-43.

59. Langille D, Rainham D, Kisely S. Is francophone language status associated with differences in the health services use of rural Nova Scotians? Can J Public Health. 2012;103(1):65-68.

60. Association canadienne de santé publique. Perceptions of health literacy : results of a questionnaire for practitioners, policymakers and researchers. Ottawa (Ont.) : Association canadienne de santé publique; 2007. PDF téléchargeable à partir du lien : http://www.cpha.ca/uploads/portals/h-l /questionnaire_e.pdf

61. Commissariat aux services en français de l'Ontario. Rapport annuel 2010-2011 : un engagement partagé [Internet]. Toronto (Ont.) : Commissariat aux services en français de l'Ontario; 2011 [consulté le 5 juin 2012]. Consultable en ligne à la page : http:// www.csf.gouv.on.ca/fr/rapport-annuel-2010 -2011-un-engagement-partage

62. Sudore RL, Landefeld CS, Pérez-Stable EJ, Bibbins-Domingo K, Williams BA, Schillinger D. Unraveling the relationship between literacy, language proficiency, and patientphysician communication. Patient Educ Couns. 2009;75(3):398-402.

63. Coleman C. Teaching health care professionals about health literacy: a review of the literature. Nurs Outlook. 2011;59(2):7078 .
64. Herndon JB, Chaney M, Carden D. Health literacy and emergency department outcomes : a systematic review. Ann Emerg Med. 2011;57(4):334-345.

65. Schlichting JA, Quinn MT, Heuer LJ, Schaefer CT, Drum ML, Chin MH. Provider perceptions of limited health literacy in community health centers. Patient Educ Couns. 2007;69(1-3):114-120.

66. Gillis D, Quigley A. Antigonish, N. S. : Taking off the blindfold : seeing how literacy affects health. Antigonish (NS) : St. Frances Xavier University; 2004. PDF téléchargeable à partir du lien : http://www.nald.ca /library/research/takngoff/takngoff.pdf

67. Kreps GL, Sparks L. Meeting the health literacy needs of immigrant populations. Patient Educ Couns. 2008;71(3):328-332.

68. Macabasco-O'Connell A, Fry-Bowers EK. Knowledge and perceptions of health literacy among nursing professionals. J Health Commun. 2011;16(Suppl.3):295-307.

69. Weiss BD, Mays MZ, Martz W et collab. Quick assessment of literacy in primary care : the newest vital sign. Ann Fam Med. 2005;3(6):514-522.

70. Hasnain-Wynia R, Wolf MS. Promoting health care equity : is health literacy a missing link? Health Serv Res. 2010;45(4): 897-903.

71. Freire P. Pedagogia da autonomia - Saberes necessários à prática educativa [Pédagogie de l'autonomie - Connaissances nécessaires à la pratique éducative]. $43^{\mathrm{e}}$ éd. São Paulo (BR) : Paz e Terra; 2011. (en portugais)

72. De Maio FG. Understanding the health transitions of immigrants to Canada: research priorities. J Health Care Poor Underserved. 2012;23(3):958-962. 